\title{
VISUAL EXTINCTION AND PHYSICAL CONDITIONS IN THE BIPOLAR NEBULA M2-9
}

\author{
A. RIERA
}

Departament de Fisica i Enginyeria nuclear, Escola Universitària de Vilanova i la Geltrú, Universitat Politécnica de Catalunya, Barcelona (Spain)

and

Departament d'Astronomia $i$ Meteorologia, Universitat de Barcelona, Av. Diagonal 647, E-08028 Barcelona (Spain)

\begin{abstract}
A detailed analysis of the visual extinction and physical conditions of the extreme bipolar nebula M2-9 has been made from optical spectra acquired with the Intermediate Dispersion Spectrograph of the $2.5 \mathrm{~m}$ Isaac Newton Telescope (Observatorio del Roque de los Muchachos, La Palma) in combination with a $235 \mathrm{~mm}$ camera and the IPCS detector covering the spectral range 3500-7500 $\AA$ with spectral resolution of $4 \AA$.

Visual extinction values through the core and the lobes have been determined from HI Balmer serie lines including self-absorption from the $2^{2}$ s metastable level. An $\mathrm{H} \alpha$ optical depth of 17 in the core and visual extinction values of 4.5 and 2.4 mag in the core and the lobes, respectively (lower than values previously estimated assuming case $\mathrm{B}$ recombination) are estimated.

Visual extinctions have also been calculated using the method developed by Allen (1979) which includes transauroral/nebular ratios of [SII] and [OII]. Values of $\mathrm{A}_{v}$ derived through the core are slightly lower than values obtained from $\mathrm{HI}$ Balmer lines, as expected if forbidden emission lines originate in an outer region. $A_{v}$ values calculated through the lobes from both methods are in agreement within the errors.

A study of the physical conditions within the nebula using dereddened fluxes of forbidden lines of $\mathrm{O}^{+}, \mathrm{O}^{++}, \mathrm{N}^{+}$and $\mathrm{S}^{+}$reveals a high stratification with a twotiered structure in which the auroral lines come from a high-density region $\left(\mathrm{n}_{e} \sim\right.$ $10^{5} \rightarrow 10^{7} \mathrm{~cm}^{-3}$ ) where nebular lines are suppresed while the nebular lines come from a low-density zone $\left(\mathrm{n}_{e} \sim 10^{3} \rightarrow 10^{4} \mathrm{~cm}^{-3}\right)$.

Both characteristics reported for M2-9: density stratification within the nebulosity and self-absorbed HI Balmer emission lines are common to symbiotic stars and some young PN. Analyses of the spectrum of M2-9 over a large spectral range (from centimeter to UV wavelengths) shows that its core shares characteristics with symbiotic stars and young PNe. However it is not well fitted by either object and could have recently evolved from one to the other as has been proposed for other bipolar nebulae such as $\mathrm{He} 2-104$ and $\mathrm{Mz} 3$.
\end{abstract}

\section{References}

Allen, D.A., 1979, MNRAS 186, 1P. 